\title{
References
}

Andrade, C. and Kurinji, S. (2002). Continuation and maintenance ECT: a review of recent research. Fournal of ECT, 18, 149-158.

Barnes, R. (2005). The use of ECT as a continuation or maintenance treatment. In A. I. F Scott. (ed.), ECT Handbook. 2nd edn. London: The Royal College of Psychiatrists.

Benbow, S. M. (1991) Old age psychiatrists' views on the use of ECT. International Fournal of Geriatric Psychiatry, 6, 317-322.

Benbow, S. M., Tench, D. W. and Darvill, S. P. (1998) Electroconvulsive therapy practice in north west England. Psychiatric Bulletin, 22, 226-229.

Lim, L.-M. (2006). A practice audit of maintenance electroconvulsive therapy in the elderly. International Psychogeriatrics, 18, 751-754.

NICE (2003). Guidance on the Use of Electroconvulsive Therapy. Technology Appraisal 59. London: National Institute for Clinical Excellence.

Pippard, J. and Ellam, L. (1981). Electroconvulsive Treatment in Great Britain, 1980. London: Gaskell.

\author{
Susan Mary Benbow ${ }^{1}$ AND DaVID TenCH ${ }^{2}$ \\ ${ }^{1}$ Professor of Mental Health \& Ageing, Centre for Ageing and Mental Health, \\ Staffordshire University, Blackheath Primary Care Trust, U.K. \\ ${ }^{2}$ Consultant Old Age Psychiatrist, Royal Oldham Hospital, Oldham, U.K.
}

doi:10.1017/S1041610207005637

\section{Three sisters covering the transient global amnesia spectrum}

We report the case of an Italian family in which three sisters experienced transient global amnesia (TGA). Since its early description, this transitory pure memory deficit has attracted increasing interest, especially within the neurological community. In 1964 the term "TGA" was coined to identify the abrupt onset of anterograde amnesia, accompanied by repetitive queries lasting for hours and then gradually recovering, leaving an amnesic gap for the duration of the attack. Afterwards, many studies focused on TGA, and in 1990 clinical criteria were defined by Hodges and Warlow (1990). Further studies showed that meeting diagnostic criteria was a significant predictor for a better outcome than in other forms of transient amnesia, while amnesic patients who did not fulfil the TGA criteria had different outcomes. Precipitating and trigger events for TGA were identified and divided into physical and psychological factors (Inzitari et al., 1997; Quinette et al., 2006). Physical precipitants were found to be gardening, housework and sawing wood, contact with water and changes in body temperature occurring during hot baths or showers, or a cold swim at the swimming pool. Emotional trigger events included a major life or death event, emotional stress triggered by a gastric endoscopy, an exhausting work session, and anxiety resulting from conflicts at home or at work, health problems and money worries. Several hypotheses have been proposed for its pathogenesis such as psychogenic, venous dysfunction due to jugular venous valve incompetence, or ischemic aetiology, but the enigma of TGA still needs to be unravelled (Lewis, 1998; Akkawi et al., 2001). 
The emerging picture of this intriguing disorder argues that TGA may be an epiphenomena triggered by different events and leading to the transitory inability to form new memories. Until now, although hundreds of cases have been reported since its first description, the family history of TGA seems to be unremarkable. Only one previous report described the history of twin sisters with migraine and TGA occurrence (Dupuis et al., 1987). Here, however, we report the case of three sisters admitted to our hospital with abrupt onset of anterograde amnesia, with repetitive queries during the TGA attack, defined according to current criteria. Despite a common familial genetic background, each one of them showed different risk factors and precipitating events, thereby underscoring a specific pathogenetic basis. Written informed consent for publication was obtained from the patients.

The first sister, aged 62 years, was admitted for TGA brought on by an argument with her husband. Memory deficits lasted for three hours, retrograde memory was preserved. No comorbidity was associated and incompetence of the jugular vein valve (JVVI) was absent. The patient described suffering severe emotional stress over the period preceding TGA and defined herself as an "anxious person." Was this a "psychogenic" TGA?

The second sister, aged 65, experienced a seven-hour attack after a cold shower. A mild hypercholesterolemia was associated and JVVI was present on the right side. Was this a transient hippocampal dysfunction due to the venous system deficits?

The third sister, aged 60, affected by hypertension and hypercholesterolemia, presented more than one attack: two episodes happened after sexual intercourse, a third after a terrifying nightmare featuring her son's death. What was the role of vascular factors in this TGA?

Previous studies found the occurrence of both emotional and physical stressful events in TGA, and showed that emotional and physical efforts and water contact/temperature change are the three most frequently observed factors associated with the personality profile of TGA patients.

TGA has been defined as a psychiatric condition, a cerebrovascular disease or a hippocampal dysfunction. Up to now, all of these theories may be supported. In fact, different phenomena - i.e. personality traits, JVVI, hypertension - may lead to a TGA attack, and may eventually co-occur even in siblings with the same genetic background. The present report further highlights this heterogeneity and the claims for a TGA spectrum rather than a unique disease.

\section{References}

Akkawi, N. M., Agosti, C., Rozzini, L., Anzola, G. P. and Padovani, A. (2001). Transient global amnesia and disturbance of venous flow patterns. Lancet; 357, 957.

Dupuis, M. J., Pierre, P. and Gonsette, R. E. (1987). Transient global amnesia and migraine in twin sisters. Fournal of Neurology, Neurosurgery and Psychiatry, 50, 816-817.

Hodges, J. R. and Warlow, G. P. (1990). The aetiology of transient global amnesia: a case control study of 114 cases with prospective follow-up. Brain, 113, 639-657.

Inzitari, D., Pantoni, L., Lamassa, M., Pallanti, S., Pracucci, G. and Marini, P. (1997). Emotional arousal and phobia in transient global amnesia. Archives of Neurology 54, 866-873. 
Lewis, S. L. (1998). Aetiology of transient global amnesia. Lancet; 352, 397-399.

Quinette, P. et al. (2006). What does transient global amnesia really mean? Review of the literature and thorough study of 142 cases. Brain; 129, 1640-1658.

Chiara Agosti, Barbara Borroni, Nabil AkKawi and

Alessandro Padovani

Department of Neurological Sciences, University of Brescia, Italy

Email: chiarett@libero.it

doi:10.1017/S1041610207005613

\section{The influence of exclusion criteria on the relationship between suicide rates and age in cross-national studies}

Traditionally, suicide rates increase with aging in many countries (Shah and De, 1998). However, exceptions to this observation are emerging. Data from the World Health Organization (WHO) in 1995 revealed that female suicide rates did not increase with age in Mauritius, Colombia, Albania and Finland (Shah and De, 1998). Suicide rates increased with age in Switzerland (AjdacicGross et al., 2006), Brazil (Mello-Santos et al., 2005) and China (Yip et al., 2000), but there were smaller peaks in the younger age-bands. Suicide rates among Australian, New Zealand and white American males increased with age, but suicide rates for females initially increased with age, peaking at menopause, and declining thereafter (Skegg and Cox, 1991; Woodbury et al., 1988; Snowdon and Snowdon, 1995). Suicide rates among non-white Americans (Seiden, 1981; Woodbury et al., 1988), Indians (Adityanjee, 1986; Bhatia et al., 1987), Jordanians (Daradekh, 1989), Indian immigrants to the U.K. (Raleigh et al., 1990; Needleman et al., 1997) and some east European countries (Sartorius, 1995) declined with increasing age.

With the emergence of studies from several countries without an increase in the suicide rate with aging, a recent cross-national study examined the relationship between suicide rates and age by obtaining data on suicide rates for males and females in seven age-bands - 16-24 years, 25-34 years, 35-44 years, 4-54 years, 55-64 years, 65-74 years and 75+ years - from the WHO website (www.3.who.int/whosis/mort/table1.cfm) for 62 countries (Shah, 2007). The main findings were: (i) a significant increase in suicide rates with increasing age in males and females in 25 and 27 countries respectively, and in both sexes in 17 countries; (ii) no significant increase in suicide rates with increasing age in males and females in 31 and 29 countries respectively, and in both sexes in 21 countries; (iii) suicide rates for all the age-bands were low in both sexes or females in countries without a significant increase in the suicide rate with increasing age in both sexes or in females only respectively; (iv) suicide rates were the highest in the younger age-bands in countries without a significant increase in male suicides rates with increasing age; (v) regional clustering was observed for countries without a significant increase in suicide rates with increasing age 